\title{
Los Criterios Descriptivo y Etiológico en la Clasificación de los Errores del Hablante no Nativo: una Nueva Perspectiva
}

\author{
Angélica Alexopoulou \\ Departamento de Lengua y Literatura Italianas y Españolas \\ Universidad de Atenas
}

Recibido: 15 marzo 2005 / Versión aceptada: 22 junio 2005

ISSN: $1697-7467$

\begin{abstract}
RESUMEN: Basados en la recopilación de un corpus de producciones escritas de aprendientes de español como lengua extranjera cuya lengua materna es el griego, reflexionamos sobre las etapas de descripción y de explicación del Análisis de Errores que representan el criterio descriptivo y etiológico respectivamente con el objetivo de aportar una contribución al perfeccionamiento de los procedimientos de análisis que poseemos.
\end{abstract}

Palabras clave: Análisis de errores. Interlengua. Descripción y explicación de errores.

\begin{abstract}
Based on the compilation of a corpus of written compositions by Greekspeaking students learning Spanish as a foreign language, we reflect upon the stage of the description and explanation of error analysis and the descriptive and explanatory criterion respectively, aiming, this way, to make a contribution to the improvement of the procedures that we possess.
\end{abstract}

Key words: Error analysis. Interlanguage. Description and explanation of errors.

\section{INTRODUCCIÓN}

Basados en la recopilación de un corpus de producciones escritas de aprendientes de español como lengua extranjera (E/LE) cuya lengua materna (LM) es el griego, reflexionamos sobre las etapas de descripción y de explicación del Análisis de Errores (AE) que representan el criterio descriptivo y etiológico respectivamente.

Estamos persuadidos de que es necesario seguir realizando estudios basados en el análisis de errores con el objetivo de identificar, describir, clasificar y explicar las producciones desviadas en la interlengua (IL) tanto escrita como oral de aprendientes de español. Dicha necesidad sigue vigente en el ámbito de E/LE, puesto que los estudios realizados hasta ahora no nos han dado una imagen representativa de las áreas de dificultad a las que se enfrentan los hablantes no nativos (HNN) de español (Penadés Martínez, 2003). Esto se hace más patente todavía si se tiene en cuenta la inexistencia de trabajos de investigación de esta índole 
en países como Grecia, a pesar del gran auge que ha experimentado el aprendizaje de E/LE durante los últimos diez años ${ }^{1}$.

Como apunta Ellis (1994: 48), el análisis de los errores no era algo desconocido para la pedagogía de las lenguas. No obstante, los estudios que se habían llevado a cabo antes de la década de los setenta carecían de una metodología y de un marco teórico que les hubiera permitido desempeñar un papel importante en la problemática de la adquisición de segundas lenguas ${ }^{2}$.

Sin perder de vista que los errores son solamente una parte de la actuación del aprendiente sobre los que se basa la descripción de su competencia transitoria, y que la otra es la que cubre la producción de formas correctas, nos adherimos a la postura de Norrish (1983: 80) que explica, de una manera simple, pero muy clara, que "un análisis de errores nos puede dar el panorama del tipo de dificultades que los aprendientes están experimentando". Pero lo que sobre todo interesa al AE es tratar de descubrir los mecanismos y las operaciones psicolingüísticas que inducen a las producciones idiosincrásicas, analizando la naturaleza, las causas y las consecuencias que éstas tienen en el proceso de aprendizaje de la L2. Las conclusiones de estos estudios pretenden contribuir a la optimización de la práctica docente.

El AE, por lo tanto, en su afán por caracterizar la IL del hablante no nativo, está constituido por dos componentes que se articulan estrechamente entre sí y que según Corder (1981: 45) son:

a) El componente teórico: como parte de la metodología de la investigación acerca del proceso de aprendizaje. Para poder descubrir la naturaleza de este proceso, nos hacen falta los medios que nos permitirán describir el sistema lingüístico no nativo.

b) El componente práctico: las conclusiones derivadas del análisis deben servir para el tratamiento pedagógico del error y, de una manera más general, deben servir de guía para el diseño de programas de estudios, la elaboración de materiales y la selección de técnicas y procedimientos de clase que, adaptados a las necesidades de los alumnos, deben incorporarse a la programación didáctica ${ }^{3}$.

En este tipo de estudios, dentro del cual se incluye el que nosotros mismos hemos llevado a cabo, los errores de los informantes representan la más valiosa fuente de información, aunque no la única, acerca de su desarrollo lingüístico a lo largo del proceso de enseñanza-

${ }^{1}$ Moreno Fernández (1995: 205-6) señala lo siguiente refiriéndose al gran número de inscripciones en los exámenes para la obtención de los Diplomas de Español como Lengua Extranjera (DELE): “destaca el número de inscritos en Grecia, país en el que la enseñanza oficial del español prácticamente no existe". Para una presentación del estado de la enseñanza de E/LE en Grecia durante los últimos años ver Cabezuela et al. (2002). Es digno de interés el dato siguiente: "El Instituto Cervantes de Atenas se encuentra entre los de mayor número de matrículas del mundo y mayor número de candidatos a los diplomas DELE”. (ibid., 23).

${ }^{2}$ Ellis cita a título indicativo los trabajos de French (1949) y de Lee (1957).

${ }^{3}$ En palabras de Corder (1973/1992: 262), el valor que tienen los errores para la clase se resumen en los siguientes puntos: "proporcionan retroalimentación, le dicen algo al maestro sobre la efectividad de sus materiales y técnicas de enseñanza, le indican qué partes del programa, que ha estado siguiendo, han sido aprendidas o enseñadas en forma inadecuada y requieren de atención adicional. Le permiten decidir si puede pasar al siguiente ítem del programa, o si debe dedicar más tiempo al ítem que ha estado trabajando [...] En términos de una planificación (sic) más amplia [...] los errores proporcionan la información para diseñar un programa de estudios que permita corregir fallos, un programa de reensñanza". 
aprendizaje de la lengua extranjera. Para la realización de un estudio como éste, es necesario seguir una metodología clara y precisa que, como instrumento de trabajo, garantice su cientificidad.

La metodología del AE procede siguiendo una serie de etapas, determinadas por primera vez por Corder (1971/1991: 72-73), quien, además, estableció las bases de la investigación del modelo de AE. Según Corder, tres son las etapas principales del $\mathrm{AE}^{4}$ :

a) La primera etapa es el reconocimiento de la idiosincrasia, en otras palabras, se trata de efectuar la identificación del error.

b) La segunda etapa es dar cuenta del dialecto idiosincrásico; esta es una actividad meramente lingüística (Corder, 1974: 128) y es conocida como la etapa de la descripción del error.

c) La tercera es la etapa que pertenece al campo de la psicolingüística y la que constituye el objetivo último del AE: nos referimos a la explicación del error.

A partir de estas etapas se pueden distinguir distintos criterios de clasificación según el aspecto desde el cual se enfoquen los errores ${ }^{5}$.

De las etapas seguidas por el AE destacan por su importancia:

- la etapa de la descripción de los errores identificados en el corpus de datos a partir del cual se procede al análisis, puesto que contempla los errores desde un punto de vista meramente lingüístico y representa el criterio descriptivo y

- la etapa de la explicación de los errores. Esta etapa se identifica con el criterio etiológico que constituye la base explicativa de nuestro estudio y es, a nuestro modo de ver, la parte más interesante y la más desafiante para el investigador, ya que descubriendo, o intentando descubrir las causas de cada error, el investigador pretende identificar los mecanismos subyacentes que conducen a un determinado comportamineto lingüístico y, por tanto, a su posible tratamiento didáctico.

La búsqueda de criterios objetivos para la evaluación de errores es uno de los problemas que plantea la investigación. La determinación de los criterios adoptados para el análisis de los errores depende de los objetivos de la investigación.

En este trabajo, en el que se lleva a cabo una clasificación de los errores registrados y un estudio cuantitativo de su frecuencia en relación con la clasificación previamente definida, intentamos establecer criterios que nos permitan agrupar y unificar los errores tanto desde la

${ }^{4}$ Estas etapas han sido completadas y ampliadas con el objetivo de que el paradigma metodológico se convierta en más operativo (Santos Gargallo, 2004: 400).

${ }^{5}$ Tanto para la determinación de los criterios como para la descripción y explicación de los errores de nuestro corpus han sido determinantes los trabajos de tres investigadoras que, en el ámbito de la lengua española, son pioneras, y gracias a las cuales se han impulsado considerablemente los estudios en el campo de la Lingüística Aplicada y más concretamente en el del AE, con su descripción exhaustiva de los mecanismos que subyacen a la construcción del sistema lingüístico no nativo. Me refiero, por orden cronológico, a los trabajos de Graciela Vázquez (1991), Isabel Santos Gargallo (1993) y Sonsoles Fernández López (1997). Estos tres trabajos, que constituyen aportaciones valiosas, nos han guiado, nos han ayudado y nos han servido de modelo. 
perspectiva del criterio descriptivo como desde la perspectiva del criterio etiológico, asignando en la medida de lo posible a cada error su fuente ${ }^{6}$.

El objetivo principal de nuestro estudio es, a partir de los datos empíricos que nos proporcionó el AE de la interlengua escrita de aprendientes adultos griegos de español, aportar una contribución al perfeccionamiento de los procedimientos de análisis que poseemos.

\section{LA DESCRIPCIÓN DE LOS ERRORES}

Toda clasificación exige una coherencia interna. Sin embargo, es muy difícil aunar concepciones ya que observamos una falta de acuerdo entre las distintas taxonomías establecidas por los varios investigadores que se han ocupado del tema (Penadés Martínez, 2003: 16).

Describir los errores implica clasificarlos para poder, a continuación, explicarlos sobre la base de los mecanismos y las estrategias que los HNN han seguido al aplicar las reglas de la gramática de su IL. Una vez pues localizados los errores, el primer paso es proceder a su clasificación según una serie de criterios claramente definidos.

Clasificar los errores significa en primer lugar diseñar un instrumento metodológico capaz de abarcar todas las categorías de los errores que pretendemos estudiar y analizar. En otras palabras, se trata de establecer una taxonomía -en este caso descriptiva- que nos permita clasificar dentro de categorías específicas todas las posibles desviaciones en la producción de nuestros alumnos.

\subsection{Criterio descriptivo}

Como hemos dicho, la descripción es una actividad puramente lingüística que involucra "varios niveles de profundidad, generalidad o abstracción" e implica explicar los errores "en términos de los procesos o reglas lingüísticos seguidos por el hablante". (Corder, 1973/1992: 273).

Teniendo como punto de partida la observación anterior, nos adherimos a la postura de Corder (op.cit., 273-4) quien mantiene que la descripción se puede hacer, por lo menos, en dos niveles:

El primer nivel y el más superficial describe los errores en términos de la diferencia física entre el enunciado del estudiante y la versión reconstruida. Las diferencias de esta clase se pueden clasificar en cuatro categorías:

a) Omisión (omission) de algún elemento obligatorio.

b) Adición (addition) de algún elemento innecesario o incorrecto.

c) Selección de un elemento incorrecto (selection of an incorrect element).

d) Mal ordenamiento de los elementos (misordering of elements).

A continuación, en un segundo nivel, podemos hacer una descripción más profunda, colocando los ítemes en cuestión en los diferentes niveles del sistema lingüístico, esto es:

${ }^{6}$ Los datos empíricos de este estudio han sido tomados de nuestra tesis doctoral inédita titulada "Contribución al estudio de los estudios morfosintácticos en la interlengua escrita de aprendientes de español de origen griego" (Universidad de Atenas, 2005). 

a) Fonético-fonológico-ortográfico
b) Morfológico
c) Sintáctico
d) Léxico-semántico
e) Discursivo
f) Pragmático

Basándonos en el primer nivel de descripción de Corder obtenemos la taxonomía del criterio descriptivo, que analizamos en lo que sigue; por otro lado, si nos basamos en el segundo nivel, obtenemos la taxonomía del criterio lingüístico ${ }^{7}$.

La primera clasificación descriptiva de Corder constituyó el marco teórico en el que se basaron otros estudiosos para definir sus propias taxonomías. Entre las más representativas citamos:

a) La de Dulay, Burt y Krashen (1982: 150-162), que proponen el término The Surface Structure Taxonomy para describir las cuatro maneras según las cuales la IL y las formas de la lengua meta divergen "de modo específico y sistemático" (omisión, adición, forma errónea y colocación falsa).

b) La de James (1998: 111), que basándose en el trabajo anteriormente citado de Dulay et al., describe las estrategias de producción según las cuales los aprendientes modifican las formas meta. Denomina su taxonomía Target Modification Taxonomy ${ }^{8}$ y la basa en una comparación de las formas que usa el HNN con aquellas que usaría el hablante nativo $(\mathrm{HN})$ en el mismo contexto. Dicha taxonomía consta de las cuatro categorías de Dulay et al., que él ha fusionado o reetiquetado (omisión, adición ${ }^{9}$, elección falsa y colocación falsa), agregando una quinta que refleja una situación de "mezcla" de dos tipos gramaticales, que se da en los casos de la denominada "asociación cruzada"10.

Tanto las categorías de Dulay et al., como las de James son similares a las de Corder. Del mismo modo, Vázquez (1991: 32-43) y Santos Gargallo (1993: 93-94) han aplicado tipologías descriptivas parecidas a las anteriores.

Tomando en cuenta estas consideraciones, en nuestro estudio la taxonomía del criterio descriptivo consta de las siguientes categorías:

a) Omisión (OM): ausencia de un morfema o de una palabra que debería aparecer. Afecta sobre todo a elementos funcionales, como por ejemplo los artículos y las preposiciones:

* espero verte _ próxima semana ${ }^{11}$

${ }^{7}$ En nuestro estudio nos hemos centrado en el nivel morfosintáctico.

${ }^{8}$ James (op. cit., 106) explica que no tiene sentido hablar de estructura superficial, ya que en este caso la dicotomía "estructura superficial vs. profunda" es inoperante.

${ }_{9}$ James denomina la adición overinclusion.

${ }^{10}$ La "asociación cruzada", a nuestro juicio, al ser una de las causas del error, forma parte del criterio etiológico y no del descriptivo.

${ }^{11}$ El asterisco delante del ejemplo indica que éste contiene una forma no gramatical. 
b) Adición (AD): presencia injustificada de un morfema o de una palabra que no debería aparecer y por lo tanto es redundante.

* un otro día

c) Elección falsa $(\mathrm{EF})^{12}$ : elección de un morfema o de una palabra incorrecta en un determinado contexto. Se trata, en la mayoría de los casos, de sustituir una unidad gramatical por otra. Es típico el ejemplo de la elección falsa de las preposiciones:

* Vivo a Atenas

d) Forma errónea (FE): uso de una forma errónea de un morfema o de una estructura en lugar de otra que sería la correcta. A nuestro modo de ver, éstos son errores típicamente formales que reflejan ignorancia o inobservancia de las reglas gramaticales. Es el caso muy frecuente de la formación errónea de las formas verbales:

* penso

e) Colocación falsa $(\mathrm{CF})$ : colocación incorrecta de un morfema o conjunto de morfemas alterando el orden sintagmático del enunciado. Estos errores suelen ser, aunque no siempre, interlinguales, ya que su causa más frecuente parece ser la interferencia. Es el caso de la alteración del orden del adjetivo:

* la civil guerra

Este nivel de descripción, como hemos dicho, es un primer paso que se sitúa en un nivel de descripción superficial. El criterio descriptivo nos ayuda a identificar los procesos cogninivos que subyacen en cada etapa del aprendizaje.

\section{LA EXPLICACIÓN DE LOS ERRORES}

El objetivo final de todo AE es la explicación de los errores. La explicación satisfactoria depende de la descripción adecuada que hayamos hecho (Corder, 1981: 36).

Se trata de inferir las causas del comportamineto lingüístico de los aprendientes, apoyándonos en la descripción de los errores efectuada previamente, formulando hipótesis acerca de los orígenes que han llevado a los HNN a producir las desviaciones en cuestión. Se intenta explicar los errores desde un punto de vista psicolingüístico con el objetivo de descifrar la lógica interna que subyace al proceso de aprendizaje.

No obstante, antes de presentar el procedimiento de esta última etapa del AE, nos parece pertinente referirnos a una de las críticas que ha recibido el AE y que alude precisamente a la relación que mantienen la descripción y la explicación. Es necesario distinguir los dos conceptos, ya que la confusión a la que se prestan puede ser motivo de problemas metodológicos. Dulay et al. (1982 : 142) clarifican el fenómeno que estamos discutiendo cuando afirman que:

- La descripción de un error se refiere al producto de la adquisición de la lengua, es el resultado del proceso de aprendizaje, que se materializa en la actuación del aprendiente. Con la descripción damos cuenta de las características superficiales de dicha actuación. errónea”.

${ }^{12}$ Dulay et al. (1982: 160) la denominan “archiform” y la consideran una subcategoría de la "forma 
- Por otro lado, la explicación del error se refiere al proceso de adquisición del lenguaje e implica la interacción entre los mecanismos internos y los factores externos que se ven involucrados. Se trata de dar cuenta de las estrategias que adopta el aprendiente y de inferir las causas que inducen al error a partir de los datos que nos proporcionó la descripción.

Las desviaciones del HNN se observan en el plano de la actuación y reflejan la violación, la sustitución o la ignorancia de una regla. La explicación de los errores intenta buscar el "cómo" y el "porqué" del comportamineto lingüístico del aprendiente mediante la observación sistemática de su producción interlingüística.

Identificar las causas del error, es decir, atribuir a cada error la causa que lo ha provocado asignándole una fuente particular, es una tarea complicada no exenta de dificultades. El investigador se enfrenta frecuentemente a errores ambiguos, que podrían atribuirse a más de una causa. A la hora de explicar las fuentes de los errores no hay que perder de vista en ningún momento que el aprendizaje de una lengua es "una interacción de factores internos y externos y la explicación de los errores debe reflejar esta interacción" (Dulay et al., 1982: 144). De ahí que que la explicación de los errores no pueda limitarse a asignar de manera unívoca cada error a una causa o fuente; este hecho dificulta la tarea del analista, que debe evitar todo tipo de juicios subjetivos.

Teniendo en cuenta esta realidad y considerando la importancia de resolver los casos ambiguos en la medida en que las circunstancias nos lo permitan, hemos tratado de ser lo más rigurosos posible en la aplicación de los criterios que hemos adoptado.

\subsection{Criterio etiológico}

El "error" suele ser el resultado de una competencia insuficiente. Cuando el alumno opta por enfrentarse a su ignorancia o conocimiento insuficiente, en vez de evitar el riesgo y guardar silencio, intenta transmitir su mensaje con los medios disponibles, activando ciertas estrategias, que, como es sabido, desempeñan un papel muy importante en la Teoría de la Interlengua, puesto que la gramática del alumno se construye paulatinamente gracias a un conjunto de reglas desarrolladas mediante estrategias cognitivas. De este conjunto de estrategias, unas conducen a producciones correctas y otras a producciones desviadas. Estas producciones desviadas de naturaleza sistemática, que evidencian la construcción de reglas por parte de los aprendientes en el proceso de construcción del nuevo sistema lingüístico, son el objeto del AE.

Nuestra intención es tratar de descubrir los principios que rigen el comportamiento lingüístico "desviado" de nuestros alumnos, reunirlos y clasificarlos con el propósito de interpretar su significado, sin olvidar que en cada situación de aprendizaje las variables contextuales e individuales pueden determinar hasta cierto punto los resultados.

Pretendemos presentar una clasificación que consiga dar a entender qué tipo de mecanismos pone en marcha el estudiante. Estamos de acuerdo con la afirmación de Jain (1974: 190) según la cual "un simple inventario de errores sin tratar de explicar su causa no revelaría nada esencial". Por otro lado, "el objetivo de cualquier AE es proporcionar una "explicación psicológica' -un informe fiable de las causas de los errores” (Abbott, 1980: 124). 
Para conseguir nuestro fin proponemos como instrumento metodológico una taxonomía de clasificación de las causas de los errores, después de pasar revista a algunas de las taxonomías más representativas que han sido propuestas a lo largo de las últimas tres décadas.

Uno de los primeros intentos por clasificar las causas de los errores es el de Selinker (1972). Selinker centra su interés en los elementos fosilizables que, según su punto de vista, son el resultado de cinco procesos cognitivos situados en la estructura psicológica latente:

1) Transferencia lingüística (positiva y negativa)

2) Transferencia de instrucción

3) Estrategias de aprendizaje

4) Estrategias de comunicación

5) Hipergeneralización de reglas de la L0

Richards (1974b), por su parte, distingue tres grandes categorías de errores:

1) Los errores interlinguales

2) Los errores intralinguales

3) Los errores evolutivos

Los primeros son excluidos de la discusión porque al autor le interesa ahondar en la reflexión de los otros dos tipos, que son los que reflejan las estrategias empleadas por el aprendiente y se derivan de la interferencia mutua de ítemes de la lengua meta (Richards, 1974b: 182). Además, ilustran algunas de las características generales de la adquisición del lenguaje. Su origen se halla, por un lado, en la estructura de la lengua en sí misma y, por otro lado, en la estrategia a través de la cual la segunda lengua ha sido adquirida y enseñada.

Los errores intralinguales se subdividen en errores causados por:

a) Hipergeneralización: es la consecuencia indeseada por aplicar indiscriminadamente el mecanismo de generalización, ${ }^{13}$ en el que se extiende, mediante una falsa generalización, el uso de una regla a casos en que ésta no se aplica, como producto de la regularización de paradigmas a partir de estructuras que se perciben como semejantes.

\section{* Quiero mejorar la idioma}

La Hipergeneralización incluye, a su vez, los errores causados por:

a1. Hipercorrección: Mecanismo contrario a la generalización, es el fenómeno en el que una excepción actúa como paradigma, en una tendencia por preferir la forma más marcada; en otras palabras, la excepción se convierte en regla.

* Te prometo que vayamos a vernos

\footnotetext{
${ }^{13}$ La generalización es una estrategia creativa mediante la cual el aprendiente intenta establecer paralelismos y analogías a partir del conocimiento adquirido previamente; es una de las operaciones fundamentales en cualquier proceso de aprendizaje y no induce al error. Lo que induce al error es la falsa generalización de una regla.
} 
a2. Sobreaprendizaje (overlearning) de una estructura: errores debidos a ciertas técnicas de instrucción, a los que nos referiremos más adelante.

b) Ignorancia de las restricciones de las reglas ${ }^{14}$ : reflejan el fracaso por parte del aprendiente para observar las limitaciones en la aplicación de una regla en contextos en que ésta no se puede aplicar. Es un mecanis mo que guarda una relación estrecha con la generalización, ya que el aprendiente se vale de su conocimiento previamente adquirido y es por esta razón que en algunos casos estos errores se pueden considerar como errores de analogía.

* Te visitaré cuando puedo

c) Aplicación incompleta de las reglas ${ }^{15}$ : Se trata de una aplicación parcial de reglas que ya existen en la IL del aprendiente; refleja el fracaso por parte del estudiante para desarrollar plenamente una estructura conocida. Tanto Norrish (1983: 32) como James (1998: 185) consideran este mecanismo el lado opuesto de la hipergeneralización, puesto que el aprendiente simplifica el sistema.

\section{* El tiempo era muy mal}

En cuanto a los errores evolutivos, son los que reflejan el intento del HNN para construir hipótesis sobre el funcionamiento de la lengua meta a partir del conocimiento limitado que posee.

Para Jain (1974) hay dos tipos de errores:

1. Los que se pueden adjudicar a la L1 (al igual que Richards los excluye de la discusión puesto que se atribuyen únicamente a la interferencia con la LM y no le interesan).

2. Los que son independientes de la L1.

Jain afirma que en cualquier situación de aprendizaje, refiriéndose tanto al niño que aprende su LM como al adulto que aprende una L2, el aprendiente tiende a reducir el lenguaje a un sistema simplificado, estrategia que se efectúa mediante la generalización. La reducción a un sistema simplificado no genera necesariamente errores. Lo hace únicamente si la reducción discrepa bastante del sistema de la lengua meta a través de la hiper-aplicación de las generalizaciones. Si, por el contrario, la reducción es selectiva, puede no generar desviaciones. Dicho de otro modo, Jain afirma que la hipergeneralización forma parte de una categoría más amplia, que viene a ser la simplificación.

Dulay y Burt (1974) y Dulay et al. (1982) clasifican los errores en cuatro categorías:

1. Errores de interferencia (1974) o interlinguales (1982): los que surgen de los problemas generados en el aprendizaje por la estructura de la L1.

${ }^{14}$ Según James (1998: 113) la ignorancia puede conducir al aprendiente a activar una estrategia, pero por sí misma no se puede considerar una estrategia.

${ }^{15}$ Bley-Vroman (1983: 6) cuestiona que la aplicación incompleta de las reglas sea una estrategia, a no ser que el aprendiente quiera ser percibido como un extranjero y para este fin pone en marcha un mecanismo consciente. 
2. Errores evolutivos: los que surgen de los problemas generados en el aprendizaje por la estructura de la L2. Son similares a los errores que cometen los niños cuando aprenden su lengua nativa. Un ejemplo típico es la hipergeneralización.

3. Errores ambiguos: son los errores que se pueden clasificar tanto como evolutivos o como interlinguales.

4. Errores únicos (1974) u otros (1982): no se pueden clasificar ni como evolutivos ni como interlinguales.

Ellis (1994) distingue entre errores de competencia y de actuación (faltas) y, señalando que se trata de desviaciones atribuibles a causas psicolingüísticas, clasifica los primeros como sigue:

1. Errores de transferencia.

2. Errores intralinguales: por ejemplo errores de hipergeneralización y los que reflejan la competencia transitoria del aprendiente.

3. Errores únicos: en los que incluye los errores inducidos.

A continuación, presentamos la categorización de James (1998). James contempla cuatro grandes categorías. Nosotros hacemos hincapié en los errores intralinguales, que él divide en siete subcategorías. La propuesta de James se resume de la manera siguiente:

1. Errores interlinguales causados por la transferencia negativa o interferencia. Se incluyen los errores generados por la traducción literal o imitación textual, sea de palabras o de relaciones gramaticales (Vázquez, 1991: 61).

2. Errores intralinguales: son generados por las estrategias de aprendizaje o de comunicación, que el aprendiente activa para enfrentar sus deficiencias. Se dividen en:
a) Falsas analogías o asociaciones cruzadas
b) Falsas hipótesis
c) Aplicación incompleta de reglas
d) Redundancia
e) Ignorancia de las restricciones (siguiendo la terminología de Richards)
f) Hipercorrección
g) Hipergeneralización o simplificación del sistema

3. Estrategias de comunicación: explican sobre todo errores léxicos.

4. Errores inducidos: ${ }^{16}$ Los aprendientes son conducidos a cometer errores por la propia naturaleza de la instrucción que reciben. Como ejemplos representativos de errores inducidos podemos mencionar:

- La explicación defectuosa de puntos gramaticales por parte del profesor.

- La presentación defectuosa de un fenómeno o de una estructura gramatical en el libro de texto.

${ }^{16}$ Con respecto a los errores inducidos, consúltese Stenson (1983). 
- La memorización de una estructura fuera de su contexto.

- Los ejercicios descontextualizados que se suelen centrar en la forma y descuidan el significado.

- La sobreproducción de algunas estructuras.

Estos errores, aunque en general no reflejan la competencia del aprendiente, es probable que se internalicen y que lleguen a formar parte de su competencia.

Por ultimo, nos parece conveniente referirnos a los criterios etiológicos presentados en dos trabajos que se han realizado en el ámbito de la enseñanza del español como lengua extranjera.

Vázquez (1987, 1991) distingue entre:

1. Los errores interlinguales

2. Los errores intralinguales

3. Los errores evolutivos que a su vez se subdividen en errores causados por:
a) Analogía
b) Hipergeneralización
c) Neutralización
d) Asociación cruzada

Fernández (1997) presenta la siguiente tipología de estrategias utilizada en la explicación de los errores:

1. Interferencia.

2. Mecanismos intralinguales: hipergeneralización o generalización

3. Analogía con estructuras próximas

4. Influencia de la forma fuerte

5. Hipercorrección

6. Neutralización de las oposiciones de la lengua meta

7. Reestructuración: aquí se incluye la estrategia de evasión o de abandono de la comunicación.

8. Formación de una hipótesis idiosincrásica

La misma autora (1997: 49) señala que todas estas estrategias se podrían resumir en dos estrategias más amplias que cubrirían las demás, esto es, la de simplificación, por un lado, y la de generalización, por otro.

Esta breve presentación de las distintas taxonomías no deja lugar a duda sobre lo complicado que es para el investigador descifrar las causas del error de manera unívoca y certera, superando las coincidencias entre las distintas categorías, los casos ambiguos y todo tipo de lagunas que se le presentan a lo largo del análisis. De todas maneras, después de este recorrido pensamos que estamos en condiciones de extraer algunas conclusiones importantes que nos guiarán en nuestra toma de decisiones, a saber: 
a) La primera gran distinción que, de manera indiscutible, podemos establecer es entre errores interlinguales y errores intralinguales.

b) Una de las mayores contribuciones del $\mathrm{AE}$ es el reconocimiento de que las causas del error pueden ir mucho más allá de la interferencia con la LM del aprendiente.

c) Los errores intralinguales, independientes de la L1 de los aprendientes, son de carácter universal y se inscriben dentro de la noción de construcción creativa por parte del HNN.

d) La hipergeneralización concebida como reorganización de los materiales linguiísticos (Richards, 1974c: 38) es una de las estrategias centrales para la construcción de la IL. Para algunos investigadores constituye, junto con la interferencia, una de las dos fuentes principales del error.

e) Aunque la simplificación podría englobar la hipergeneralización, hemos decidido, por razones metodológicas, considerarla como una estrategia aparte. En general, uno de los rasgos que diferencian estas dos estrategias es que la primera opera a partir de la reducción de los elementos que se consideran redundantes, con una tendencia clara hacia las formas menos marcadas y por lo tanto más universales y más frecuentes, mientras que la segunda opera a partir del conocimiento previo para aplicar las reglas más allá de su dominio sin tener en cuenta sus restricciones; en algunas ocasiones, como es el caso de la hipercorrección, se prefieren las formas más marcadas, es decir, las más periféricas y menos frecuentes ${ }^{17}$.

Después de cotejar las tipologías que hemos presentado y tomando en cuenta los objetivos y la naturaleza de nuestro trabajo, proponemos en la Figura 1 una tipología de estrategias, que constituye el criterio etiológico que hemos utilizado en nuestro estudio para explicar las causas de los errores morfosintácticos que hemos detectado en nuestro corpus. Estas estrategias del aprendiente, a partir de las cuales se generan tanto los errores interlinguales como los intralinguales, consideramos que son estrategias compensatorias, pertenecientes a las estrategias de comunicación (Faerch y Kasper, 1983: 53) y resultan de la imposibilidad para compensar las deficiencias a la hora de hacer frente a una situación comunicativa.

Por último, nos parece importante comentar que, aunque en nuestro corpus no la hemos rastreado, hemos integrado la estrategia de evasión tanto en los errores interlinguales como en los errores intralinguales considerándola como una de las principales estrategias de comunicación (Faerch y Kasper, 1983; Tarone, 1981). A nuestro parecer, la evasión en cuanto sustitución de la forma con la que el estudiante se siente inseguro por otra con la que se siente más familiarizado, puede dar lugar a errores de los dos tipos. No nos referimos aquí a la evasión del contenido del mensaje, que generaría sobre todo errores léxicos, ni a la variante extrema de la evasión que conduce al abandono del mensaje y, por lo tanto, al no error.

${ }^{17}$ Acerca de la Hipótesis de la Marca Diferencial véase Eckman (1977/1991). 
Figura 1. Criterio etiológico.

\begin{tabular}{|l|l|}
\hline \multicolumn{2}{|c|}{ CRITERIO ETIOLÓGICO } \\
\hline \multirow{3}{*}{ ERRORES INTERLINGUALES } & - INTERFERENCIA \\
& - Interferencia de la L1 (IF) \\
& - Interferencia de otra L3 \\
& - Traducción literal \\
& - Evasión \\
\hline \multirow{5}{*}{ ERRORES INTRALINGUALES } & - REDUCCIÓN A UN SISTEMA SIMPLIFICADO \\
& - Simplificación (SPL) \\
& - Neutralización (NTR) \\
& - Eplicación incompleta de las reglas (AIR) \\
\cline { 2 - 3 } & - GENERALIZACIÓN \\
& - Hipergeneralización (HG) \\
& - Analogía (AN) \\
& - Asociación cruzada (ACr) \\
& - Ignercorrección (HC) \\
& (IRR) \\
\hline
\end{tabular}

\section{Aplicación de los Criterios descriptivo y etiológico a un corpus DE ERRORES MORFOSINTÁCTICOS}

Presentamos a continuación los resultados que nos proporcionó el análisis de errores que hemos efectuado a partir de una muestra constituida por un corpus de datos compuesto de 98 pruebas de producción escrita pertenecientes a alumnos adultos del Instituto Cervantes de Atenas y agrupadas de acuerdo con los cuatro niveles de evolución, a saber, A (inicial), B (medio), C (avanzado) y D (superior).

En cuanto a la técnica de recogida de datos, fue determinada, no sólo por el tipo de habilidad lingüística (expresión escrita), sino también por los objetivos que nos hemos fijado. Dos son los puntos que nos parece importante valorar: el primero es que es imposible estudiar todos los aspectos del desarrollo interlingüístico de los informantes. El segundo es que los propios informantes establecen limitaciones en los datos que proporcionan (Corder, 1981: $60)$.

El procedimiento del que nos hemos valido es el de redacción libre, con un tema asignado por el Instituto Cervantes, con diversas opciones (a excepción del nivel A). La posibilidad de seleccionar un tema nos permite intuir que el alumno habrá elegido aquél con el que tenía la impresión de sentirse más a gusto. Por otro lado, hemos optado por la redacción libre, puesto que es el procedimiento que ejerce el mínimo control sobre la actuación (LarsenFreeman, 1991/1994: 39). Es decir, no se trata de un ejercicio de producción guiada, en el sentido de orientar a los aprendientes a que produzcan la estructura que nos interese, porque 
nuestro propósito no era el de estudiar un fenómeno gramatical en concreto, sino localizar todo tipo de error morfosintáctico. La redacción libre, por consiguiente, es una técnica de recogida de datos que "los obliga" a utilizar todos los medios lingüísticos de los que disponen.

Con respecto a la periodicidad en la recogida de datos, valorando la viabilidad de nuestra investigación, hemos optado por un estudio seudolongitudinal que se aproxima al análisis de la interlengua en intervalos periódicos, pero con informantes diferentes en cada uno de los estadios objeto de observación.

El total de las 1524 producciones erróneas que nos proporcionó el corpus de nuestros informantes nos ha permitido rastrear áreas particulares de dificultad en el nivel morfosintáctico. La interpretación de estos datos nos permite indagar sobre las causas y el origen de los errores, con el objetivo de detectar las estrategias comunicativas aplicadas por parte de los aprendientes a la hora de emitir un mensaje en la lengua meta. En la Figura 2 se ofrece una visión global de las estrategias empleadas en el conjunto de los cursos.

Figura 2. Criterio etiológico (visión global).

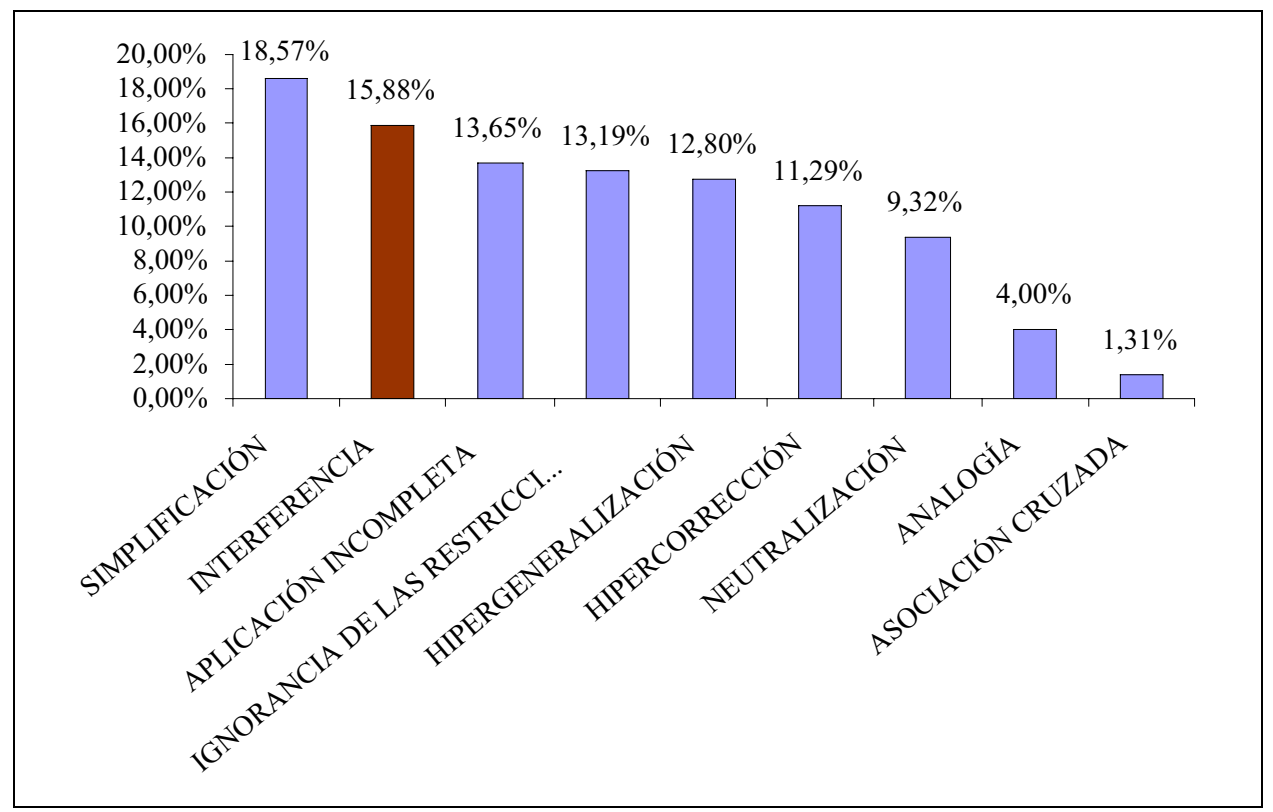

La comparación de cada una de las estrategias con las categorías del criterio descriptivo, nos dio los resultados que ofrecemos en el siguiente cuadro (Figura 3): 
Figura 3. Relación entre criterio etiológico y criterio descriptivo.

\begin{tabular}{|l|c|c|c|c|c|c|c|c|c|c|}
\hline \multirow{2}{*}{$\begin{array}{l}\text { CRITERIO } \\
\text { DESCRIPTIVO }\end{array}$} & \multicolumn{10}{|c|}{ CRITERIO ETIOLÓGICO } \\
\cline { 2 - 12 } & IF & SPL & NTR & AIR & HG & AN & HC & ACr & IRR & TOTAL \\
\hline AD & 45 & 1 & - & & 18 & 1 & 101 & 11 & 9 & 186 \\
\hline OM & 7 & 172 & 4 & 84 & 3 & - & - & & 1 & 271 \\
\hline EF & 90 & 49 & 138 & 66 & 131 & 11 & 52 & 4 & 150 & 691 \\
\hline FE & 77 & 61 & - & 52 & 43 & 49 & 17 & 5 & 13 & 317 \\
\hline CF & 23 & - & - & 6 & - & - & 2 & - & 28 & 59 \\
\hline TOTAL & 242 & 283 & 142 & 208 & 195 & 61 & 172 & 20 & 201 & 1524 \\
\hline
\end{tabular}

Como muestran las cifras, la categoría que destaca en el criterio descriptivo es la elección falsa, que resulta ser mayoritaria en casi todas las estrategias. La gráfica (Figura 4) que presentamos nos permite apreciar de manera global los resultados obtenidos en relación con el criterio descriptivo en el conjunto de los niveles tratados. Contrastándolos con los resultados de cada nivel por separado, comprobamos que la proporción no cambia.

Figura 4. Criterio descriptivo (visión global).

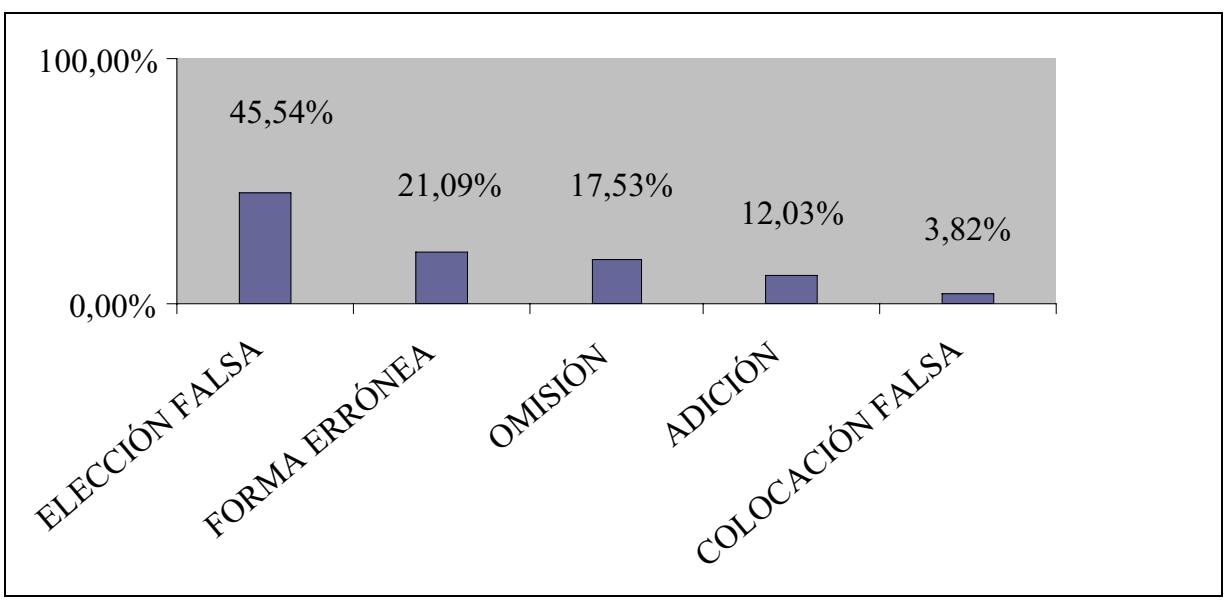
puntos:

Del mismo modo, se pueden verificar algunas constantes, que se concretan en los siguientes 
a) Los errores de interferencia (IF) son el producto, sobre todo, de la elección falsa (EF) y de la forma errónea (FE) de algún ítem de la L2.

b) Los errores de simplificación (SPL) se producen mayoritariamente por la omisión (OM) de algún elemento.

c) Los errores de neutralización (NTR) provienen casi todos de la EF de estructuras, que suelen encontrarse en distribución complementaria.

d) Los errores de aplicación incompleta de las reglas (AIR) pueden generarse tanto a partir de la OM como a partir de la EF o de la FE.

e) Los errores de 'hipergeneralización (HG), en su gran mayoría, son el resultado de una EF.

f) Los errores de analogía (AN) son el producto de la FE.

g) Los errores de hipercorrección (HC) resultan, en la mayor parte de los casos, de la adición (AD) de algún elemento, puesto que esta estrategia lleva a utilizar la forma más "difícil" en casos en los que no es necesario.

h) La asociación cruzada ( $\mathrm{ACr}$ ) es también mayormente el producto de alguna AD.

i) La ignorancia de las restricciones de las reglas (IRR) es igualmente el resultado de la EF.

De los datos expuestos anteriormente y contrastando los resultados, podemos afirmar que se comprueban las siguientes tendencias:

a) Los errores interlinguales, esto es los que se originan por interferencia con la LM y, en menor proporción con otra L3, representan apenas una sexta parte en el conjunto de los errores de nuestro corpus o un porcentaje de 15,9\% en el total de las desviaciones.

b) Los errores intralinguales representan, en el conjunto de las producciones erróneas, un promedio del $82,7 \%$.

c) De las estrategias intralinguales, la estrategia más empleada es la simplificación que lleva al aprendiente a reducir elementos de la lengua meta al percibirlos como redundantes; seguida por la aplicación incompleta de las reglas, por la ignorancia de las restricciones de las reglas aprendidas, pero todavía no interiorizadas del todo; y por la hipergeneralización, que conduce a la aplicación de una regla aprendida más allá de su dominio.

d) Los errores intralinguales que, en general, responden a un dominio incompleto de la lengua meta, se pueden dividir en dos grandes categorías:

d1) Los errores de simplificación del sistema, que incluye, además de la simplificación en sí, los errores generados por la neutralización y por la aplicación incompleta de las reglas, que representan en total el $40 \%$ de las desviaciones.

d2) Los errores de generalización, que incluyen los errores de hipergeneralización, de hipercorrección, de ignorancia de las restricciones de las reglas, de analogía y de asociación cruzada, representan en total el 42,8\% de los producciones erróneas.

e) Las dos grandes subcategorías que constituyen los errores interlinguales comparten casi el mismo porcentaje y el conjunto de las dos subcategorías constituye la inmensa mayoría de las causas que pueden explicar los errores cometidos por nuestros informantes. 
f) En cuanto a la evolución de los errores intra e interlinguales que se pueden apreciar en la Figura 5, observamos que los errores interlinguales empiezan a disminuir levemente a partir del nivel $\mathrm{C}$, que junto con el nivel $\mathrm{B}$ es el que presenta el mayor porcentaje de errores causados por interferencia, siguiendo una curva descendente para llegar al nivel D, donde observamos el porcentaje más reducido. Este fenómeno es comprensible si se tiene en cuenta que en los primeros niveles, y sobre todo en el segundo, los alumnos, sintiéndose más inseguros, recurren de manera más frecuente a su LM.

g) Los errores intralinguales, por su parte, siguen el proceso contrario, puesto que, como se puede observar, a partir del nivel B empieza a ascender la curva evolutiva hasta llegar a su mayor grado en el nivel D, momento en el cual los HNN sienten que han adquirido una competencia lingüística que se aproxima a la de los HN, motivo por el cual pueden independizarse más de su LM.

Figura 5. Evolución de los errores intra e interlinguales.

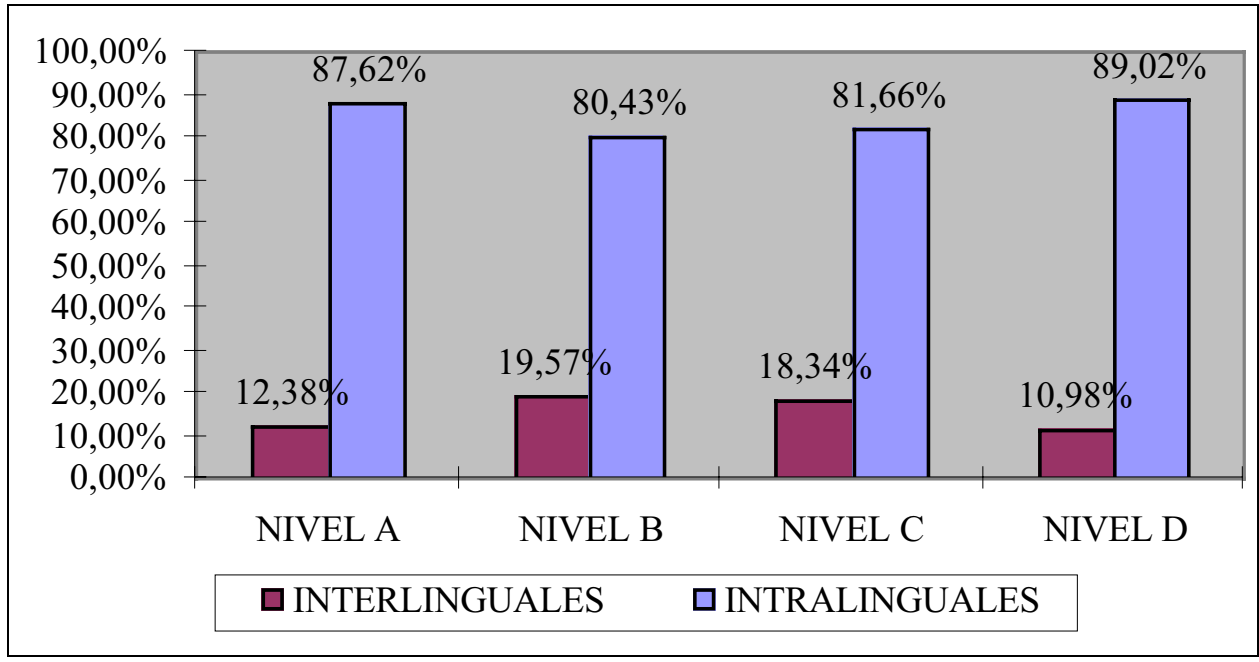

\section{Conclusiones}

En este estudio nos propusimos indagar sobre las causas de las producciones desviadas de nuestros informantes con el fin de contribuir al descubrimiento de los principios psicolingüísticos que intervienen en el proceso de aprendizaje de E/LE. El objetivo final del $\mathrm{AE}$ es, a partir de los resultados obtenidos, ofrecer a los profesores de E/LE una serie de pautas que los guien en el diseño de programas de estudio, en la elaboración de materiales y actividades didácticas y en la selección de técnicas y procedimientos de clase de acuerdo con las necesidades del alumno, a fin de optimizar la práctica docente. Desde este punto de vista se hace patente la necesidad de realizar estudios específicos encauzados a ofrecer el 
marco en el que se apoyaría la confección de materiales didácticos y el diseño de planes curriculares.

Esperamos que este primer estudio, muy lejos de agotar la problemática en la que está inscrito, haya contribuido mínimamente a revelar la necesidad de profundizar en los aspectos de la adquisición de E/LE en relación con los aprendientes cuya lengua nativa es el griego. Esta ha sido por lo menos nuestra modesta intención.

\section{REFERENCIAS BIBLIOGRÁFICAS}

Abbott, G. (1980). “Towards a More Rigorous Analysis of Foreign Language Errors”, en International Review of Applied Linguistics, 18: 121-34.

Bley-Vroman, R.W. (1983). "The Comparative Fallacy in Interlanguage Studies: The Case of Systematicity", en Language Learning, 33: 1-13.

Cabezuela, M, Maresma, J. y Maestro, O. R. (2002). "La situación del español en Grecia", en Boletín de la Asociación para la Enseñanza del Español como Lengua Extranjera, 26: 2328.

Corder, S. P. (1971). "Idiosyncratic Dialects and Error Analysis", en International Review of Applied Linguistics, 9,2: 147-60. (Traducción al español: "Dialectos idiosincrásicos y análisis de errores", en J. Muñoz Liceras (comp.) (1991), La adquisición de las lenguas extranjera. Madrid: Visor, 63-77)

Corder, S. P. (1973). Introducing Applied Linguistics. Harmondsworth: Penguin. (Traducción al español: (1992). Introducción a la lingüística aplicada. México: Limusa).

Corder, S. P. (1974). "Error Analysis", en J.P Allen y S.Pit Corder (eds.), The Edinburgh Course in Applied Linguistics. Vol.3. Techniques in Applied Linguistics. London: Oxford University Press. 122-154.

Corder, S.P. (1981). Error Analysis and Interlanguage. Oxford: Oxford University Press.

Dulay, H.C. y Burt. M.K. (1974)."You Can't Learn Without Goofing. An Analysis of Children's Second Language 'Errors' en. J. Richards (ed.),nError Analysis: Perspectives on Second Language Acquisition. London: Longman,. 95-123.

Dulay, H., Burt, M. y Krashen, S. (1982).nLanguage Two. $n$ New York: Oxford University Press. Eckman, F. (1977). "Markedness and the Contrastive Analysis Hypothesis", en Language Learning, 27,2: 315-330. (Traducción al español: "El análisis contrastivo y la teoría de lo marcado", en J. Muñoz Liceras (comp.) (1991),“La adquisición de las lenguas extranjera. Madrid: Visor, 207-224).

Ellis, R. (1994). The Study of Second Language Acquisition. Oxford: Oxford University Press.

Faerch, C. y Kasper G. (1983). "Plans and Strategies in Foreign Language communication", en C. Faerch y G. Kasper (eds.), Strategies in Interlanguage Communication. London: Longman, 20-60.

Fernández López, S. (1997). Interlengua y análisis de errores en el aprendizaje del español como lengua extranjera. Madrid: Edelsa.

Jain, M.P. (1974). "Error Analysis: Source, Cause and Significance", en J. Richards (ed.), Error Analysis: Perspectives on Second Language Acquisition. London: Longman, 189-215.

James, C. (1998). Errors in Language Learning and Use. London: Longman.

Larsen-Freeman, D. y Long, M. (1991). An Introduction to Second Language Acquisition Research. Londres: Longman. (Traducción al español: (1994). Introducción al estudio de la adquisición de segundas lengua. Madrid: Gredos). 
Moreno Fernández, F. (1995). "La enseñanza del español como lengua extranjera", en Marqués de Tamarón (dir.), El peso de la lengua española en el mundo. Valladolid: Secretariado de Publicaciones de la Universidad de Valladolid, 195-233.

Norrish, J. (1983). Language Learners and their Errors. London: Macmillan Press.

Penadés Martínez, I. (2003). "Las clasificaciones de errores lingüísticos en el marco del análisis de errores", en Lingüística en la red, Universidad de Alcalá. [http://www2.uah.es/linred/ articulos pdf/LR articulo 051120032.pdf]

Richards, J.C. (1974a). Error Analysis: Perspectives on Second Language Acquisition. London: Longman.

Richards, J.C. (1974b). “A Non-Contrastive Approach to Error Analysis”, en J. Richards (ed.), Error Analysis: Perspectives on Second Language Acquisition. London: Longman, 172187.

Richards, J.C. (1974c). "Error Analysis and Second Language Strategies", en J. Schumann \& N. Stenson (eds.), New Frontiers in Second Language Learning. Rowley, Massachusetts: Newbury House Publishers, 32-51.

Santos Gargallo, I. (1993). Análisis Contrastivo, Análisis de Errores e Interlengua en el marco de la Lingüística Contrastiva. Madrid: Síntesis.

Santos Gargallo, I. (2004). "El análisis de los errores en la interlengua del hablante no nativo", en Sánchez Lobato J. e I. Santos Gargallo (dir), Vademécum para la formación de profesores. Madrid: SGEL, 391-410.

Selinker, L. (1972). "Interlanguage”, en"International Review of Applied Linguistics, 10.3: 209-31. (Traducción al español: "La interlengua”, en J. Muñoz Liceras (comp.) (1991), La adquisición de las lenguas extranjera. Madrid: Visor, 79-101).

Stenson, N. (1983). "Induced Errors", en B. W. Robinett and J. Schachter (eds.), Second Language Learning: Contrastive Analysis, Error Analysis and Related Aspects. Ann Arbor: University of Michigan Press, 256-271.

Tarone, E. (1981). "Some Thoughts on the Notion of Communication Strategy", en TESOL Quarterly, 15, 3: 285-295.

Vázquez, G. (1987). "Hacia una valoración positiva del concepto del error", en Actas de las Jornadas Internacionales del E-LE. Madrid: Ministerio de Cultura, 151-162.

Vázquez, G. (1991). Análisis de errores y aprendizaje de español lengua extranjera. Análisis, explicación y terapia de errores transitorios y fosilizables en el proceso de aprendizaje de español como lengua extranjera en cursos universitarios para hablantes nativos de alemán. Berlin: Peter Lang. 